\title{
Non-unitary Scattering Theory against Generalized
}

\section{Unitary Scattering Theory}

\author{
Jacek A. Jakiel and Wiesław A. Kantor \\ Institute of Nuclear Physics Polish Academy of Sciences, PL-31342 Krakow, Poland
}

\begin{abstract}
Comparison of non-unitary and generalized unitary scattering theories is done by means of nuclear monodromy (equivalence of Schrödinger and Maxwell time-independent equations), tunneling and radioactivity. Radioactivity is important part of physics and our life. Its importance stretches from medicine as far as to war strategies. We present theoretical approach to achieve better understanding of the radioactive decay when modified quantum theory is applied. It can be done by updating existing codes to understand better construction of the world and terms and conditions of our existence. The theory modifications are strictly connected with the unimodular M matrix and Wronskian matrices (i.e. their determinants named Wronskians) which create underpinning of so called monodromy being two track wave-function evolution.
\end{abstract}

Key words: Nuclear monodromy, radioactivity, scattering theory, unitarity, tunneling, boundary condition.

\section{Introduction}

In mathematical physics, scattering theory helps to understand collision of waves and particles with some material object. The scattering of the light has a long history and has begun when Aristotle (384322 BC) tried to explain the rainbow formation. Then in the next centuries, many scientists (like Descartes, Newton and many others) attempted to improve Aristotle's qualitative description but without any significant success until Thomas Young (1773-1829) optical wave theory. Further developments can be found e.g. in Ref. [1].

In whole XX century wave-particle duality had caused fierce discussions about quantum mechanics interpretation with emphasis on philosophical application rather than on new mathematical solutions of the Schrödinger equation. It was also battle between classical and quantum mechanics. Heisenberg developed the matrix mechanics starting from classical Hamiltonians and was unable to accept the Schrödinger point of view.

That battle has led to simplification and

Corresponding author: Jacek A. Jakiel, Ph.D., research field: nuclear physics (scattering theory). reconciliation of those different issues (quantum and classical, wave and particle). Due to such way of thinking we have no quantum spin model and quantum scattering theories are unitary and reversible.

The simplest model of two particle reversible classical collision is interaction of two balls on the billiard table in the center of their mass system. In analogy, in the nuclear physics we speak about detailed balance or micro reversibility relation expressed by the unitary $S$ matrix as: $\boldsymbol{S}_{f \leftarrow i}=\boldsymbol{S}_{i \leftarrow f}$.

What seems to be missing in such theoretical consideration of scattering processes? Due to simplified boundary condition, quantum collision is built in analogy to the balls collision. The particles can be reflected but tunneling-transmission is prohibited.

To include reflection and tunneling-transmission, generalization of $S$ matrix can be done according to Wronskian rules determining the matrix two channel solutions of the Schrödinger equation. There are two ways to challenge above problem:

(a) We can upgrade the standard scattering theory by means of modified partial waves method and reversibility is prevented, or 
(b) We can solve the Schrödinger equation according to Wronskian rules [2] determining the monodromy matrix two channel solutions of the Schrödinger equation losing reversibility.

Ad. (a): In the asymptotic forms of the Schrödinger equation solution (in principle, plane wave plus an outgoing wave) $\psi_{\vec{k}}(\vec{r}) \underset{r \rightarrow \infty}{\longrightarrow} e^{i k z}+f(\vartheta) \frac{e^{i k r}}{r}$, we replace the standard scattering amplitude $f(\vartheta)$ with two amplitudes $\boldsymbol{f}_{\boldsymbol{R}}(\boldsymbol{\vartheta}), \boldsymbol{f}_{\boldsymbol{T}}(\boldsymbol{\vartheta})$ suitably for reflection and transmission processes according to the probability condition:

$$
|\boldsymbol{R}|^{2}+|\boldsymbol{T}|^{2}=1
$$

Then the asymptotic form of the modified scattering wave function is

We define the partial amplitudes:

$$
\psi_{\vec{k}}(\vec{r}) \underset{r e f l, t r a n s, r \rightarrow \infty}{\longrightarrow} e^{i k z}+f_{R}(\vartheta) \frac{e^{-i k r}}{r}+f_{T}(\vartheta) \frac{e^{i k r}}{r} \equiv e^{i k z}+R f(\vartheta) \frac{e^{-i k r}}{r}+T f(\vartheta) \frac{e^{i k r}}{r}
$$

$$
\begin{aligned}
& R_{l}^{f}=R_{l} f_{l}(\vartheta) \\
& T_{l}^{f}=T_{l} f_{l}(\vartheta)
\end{aligned}
$$

Then the partial waves expansion looks like:

$$
\begin{aligned}
& \boldsymbol{k} \boldsymbol{r} \psi_{\overrightarrow{\boldsymbol{k}}}=\sum_{\boldsymbol{l}} \frac{2 \boldsymbol{l}+1}{2} \boldsymbol{i}^{l}\left[\left(1+\boldsymbol{i} \boldsymbol{T}_{\boldsymbol{l}}{ }^{f}\right) \mathbf{H}_{\boldsymbol{l}}^{\left({ }^{(1)}\right.}(\boldsymbol{k r})+\left(1+\boldsymbol{R}_{\boldsymbol{l}}{ }^{f}\right) \mathbf{H}_{l}{ }^{(2)}(\boldsymbol{k r})\right] \boldsymbol{P}_{\boldsymbol{l}}
\end{aligned}
$$

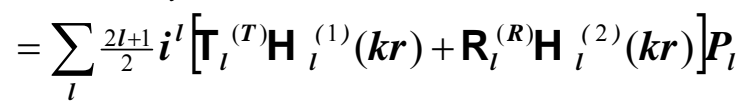

where $\mathbf{H}_{\boldsymbol{l}}$ are Hankel $\mathbf{H}_{\boldsymbol{l}}=\boldsymbol{j}_{\boldsymbol{l}} \pm \boldsymbol{n}_{\boldsymbol{l}}$ or Coulomb $\left(\mathbf{H}_{\boldsymbol{l}}=\boldsymbol{F}_{\boldsymbol{l}} \pm \boldsymbol{i} \boldsymbol{G}_{\boldsymbol{l}}\right)$ wave functions, see Section 2 and footnote 2 there.

Now we introduce auxiliary matrices: transmission one $S_{I}^{T}$ and another reflection- transmission $S_{I}^{(r t)}$ :

$$
\begin{gathered}
S_{I}^{T}=\frac{1+i T_{l}^{f}}{N_{T}}=\frac{1+i T_{I} f_{l}}{\sqrt{\left|1+i T_{I}^{f}\right|^{2}}} \\
S_{l}^{(r t)}=R_{I}-i T_{I}
\end{gathered}
$$

With the reflection-transmission $S_{I}^{(r t)}$ matrix the partial waves expansion takes the following form:

$$
\boldsymbol{k r} \psi_{\vec{k}}(\overrightarrow{\boldsymbol{r}})=\sum_{l} \frac{2 l+1}{2} \mathbf{i}^{l} \boldsymbol{N}_{T} \boldsymbol{S}_{I}^{T}\left[\mathrm{H}_{l}^{(1)}(\boldsymbol{k r})+\mathbf{S}_{l} \mathbf{H}_{l}{ }^{(2)}(\boldsymbol{k r})\right] \boldsymbol{P}_{I}
$$

Where $\mathbf{S}_{\boldsymbol{I}}$ is the redefined new unitary matrix

$$
\mathbf{S}_{\mathbf{I}}=\mathbf{R}_{I}^{\left(R^{(R)}\right.} / \mathbf{T}_{I}^{(T)}=\left(1+\frac{\boldsymbol{R}_{I}^{f}-i T_{I}^{f}}{N_{T} \frac{1+i i_{I}^{f}}{N_{T}}}\right)=\left(1+\frac{R_{I}-i T_{I}}{N_{T} S_{I}^{T}} 2 i f_{I}\right)=S_{I}^{(T)-1}\left(S_{I}^{T}+S_{I}^{(r t)} \frac{2 i f_{I}}{N_{T}}\right)=S_{I}^{(T)-1} S_{I}
$$

On the other hand comparing one point and two points boundary condition and considering monodromy as a base, we modify the scattering theory to include tunneling. Then, as it is shown above (the modification of the optical theorem is sketched using the partial wave method applied to the scattering-tunneling theory) the optical theorem becomes changed too. After taking into account two amplitudes $\boldsymbol{f}_{R}(\boldsymbol{\vartheta}), \boldsymbol{f}_{T}(\vartheta)$ the imaginary part of the total forward scattering amplitude turned around in R, T space (i.e. $\left.\rightarrow\left(\boldsymbol{f}_{\boldsymbol{R}}, \boldsymbol{f}_{\boldsymbol{T}}\right) \times\left.(\boldsymbol{R}, \boldsymbol{T})\right|_{\mathbf{z}}\right)$ is equal to the total cross section diminished by the transmission crosssection.

$$
\frac{4 \pi}{k} \sum_{\boldsymbol{l}}\left(\boldsymbol{R}_{\boldsymbol{l}} \operatorname{Im} \boldsymbol{f}_{\boldsymbol{l}}-\boldsymbol{T}_{\boldsymbol{I}} \operatorname{Re} \boldsymbol{f}_{\boldsymbol{l}}\right)=\int|\boldsymbol{f}(\Omega)|^{2} \boldsymbol{d} \Omega-\sum\left|\boldsymbol{T}_{\boldsymbol{l}}\right|^{2}\left|\boldsymbol{f}_{\boldsymbol{I}}\right|^{2}=\int|\boldsymbol{f}(\Omega)|^{2} d \Omega-\int\left|\boldsymbol{f}_{T}(\Omega)\right|^{2} d \Omega
$$


The similar but simplified analysis can be found in Ref. [3].

For the pure reflection $R=1 ; T=0$ we recover the classical optical theorem, while including transmission i.e. $\boldsymbol{T} \neq 0$, the total cross section is diminished by the transmission-tunneling cross section what constitutes the base of the generalized Ramsauer-Townsend effect $[4,5]$.

Two point boundary conditions enable reanalysis of the Gamow theory using the Wronskian solutions of the Schrödinger equation i.e. with tunneling through the barrier being taken into consideration.

Summing up, when holding on to unitarity this work becomes typical scattering theory overlay, if applied to Joachain's monograph [6]. Many formulas in Ref. [6] when monodromy is applied, require generalization.

People used to say that the direct scattering problem is the problem of determining the distribution of scattered flux basing on the characteristics of the scatterer while the inverse scattering determines characteristics of an object i.e. its structure. The direct scattering analysis is possible if we assume correctness of the theoretical model (here: the optical model).

Ad. (b): A new solution of the Schrödinger equation which enables us to test influence of the barrier shape on the measured quantities in scattering experiments is presented in the next section. Instead of the simplified probability condition (1) we work with the condition which depends on directions (left $\rightarrow$ right or right $\rightarrow$ left) and reversibility is lost:

$$
\left|\boldsymbol{R}_{l}^{(f, i n)}\right|^{2}+\left|\boldsymbol{T}_{l}^{(f, i n)}\right|^{2}=\left|\boldsymbol{R}_{l}^{(i n, f)}\right|^{2}+\left|\boldsymbol{T}_{l}^{(i n, f)}\right|^{2}
$$

If the following relation is kept

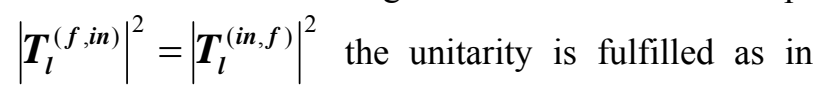
item (a).

\section{Quantum Mechanics Gamow Decay}

Detailed analysis of Gamow decay allows us to understand how reflection and transmission amplitudes influence the standard optical model. What is the cause that radioactive decay is not included in the optical model? To understand differences we examine the boundary condition put on the S-matrix formation.

In the standard scattering theory the following boundary conditions are applied:

$$
\begin{aligned}
& u(0)=0 \\
& \beta_{\psi} u(b)+\beta_{\psi^{\prime}} u^{\prime}(b)=0
\end{aligned}
$$

We stress the wave function's equality to zero at the origin of coordinate system i.e. at the nucleus center, is rather the artificial action and cannot be explained by potential singularity at that point (where Coulomb potential tends to infinity). In the optical model's calculations considered as standard methods of cross section analysis, the Schrödinger equation's integration starts ${ }^{1}$ with the regular solution $\boldsymbol{u}_{1}(\boldsymbol{r})$ at the system origin. The matching procedure resulting in the phase shifts shows contribution of the irregular wave function $\boldsymbol{u}_{2}(\boldsymbol{r})$ :

The regular solution is:

$$
\begin{aligned}
& \boldsymbol{u}_{1}(\boldsymbol{r}) \stackrel{r \rightarrow \infty}{\longrightarrow} \frac{1}{2}\left\{\boldsymbol{F}_{\boldsymbol{l}}+\mathbf{i} \boldsymbol{G}_{\boldsymbol{l}}+\boldsymbol{S}_{\boldsymbol{l}}\left(\boldsymbol{F}_{\boldsymbol{l}}-\mathbf{i} \boldsymbol{G}_{\boldsymbol{l}}\right)\right\}= \\
& =F_{l}+C_{l}\left(G_{l}+i F_{l}\right) \text { : } \\
& =e^{i \eta_{I}}\left(F_{l} \cos \eta_{I}+G_{I} \sin \eta_{l}\right)
\end{aligned}
$$

Here $C_{l}=\frac{1}{2 i}\left(S_{l}-1\right)=\boldsymbol{e}^{i \eta_{I}} \sin \eta_{\boldsymbol{l}}$ are the scattering coefficients; $S_{I} \equiv \boldsymbol{e}^{2 i \eta_{I}}=1+\mathbf{T}_{\ell}=1+2 \mathbf{i} C_{I}$ is the scattering matrix element. Usually the regular solution can be written as superposition of the inward function $\mathbf{H}^{\left({ }^{1}\right)}(\boldsymbol{k r})=\mathbf{H}^{\boldsymbol{i n} \boldsymbol{w}}(\boldsymbol{k r})$ (i.e. Coulomb function, Hankel etc.) and outward one ${ }^{2}$ $\mathbf{H}^{\left({ }^{2}\right)}(\boldsymbol{k r})=\mathbf{H}^{\text {outw }}(\boldsymbol{k r})$.

$$
\begin{aligned}
& u_{1}(r)=u(r)= \\
& r \psi_{k}(r) \stackrel{r \rightarrow \infty}{\longrightarrow} \frac{1}{2}\left\{H^{i n w}(k r)+S H^{\text {outw }}(k r)\right\}
\end{aligned}
$$

The irregular solution can be written as:

$$
\boldsymbol{u}_{2}(\boldsymbol{r})=\boldsymbol{e}^{i \eta_{l}}\left(\boldsymbol{G}_{\boldsymbol{l}} \cos \eta_{l}-\boldsymbol{F}_{\boldsymbol{l}} \sin \boldsymbol{\eta}_{l}\right)
$$

and in practice is not taken directly into consideration. It modifies only $\boldsymbol{u}_{1}(\boldsymbol{r})$ evolution through the nuclear 
phase shifts coefficients $\boldsymbol{C}_{\boldsymbol{l}}$.

The standard scattering theory deals with one-track solution but not with two-track $\boldsymbol{u}_{1}(\boldsymbol{r}), \boldsymbol{u}_{2}(\boldsymbol{r})$ solutions. According to boundary conditions, $\boldsymbol{u}(\boldsymbol{r})=\boldsymbol{r} \boldsymbol{\psi}_{\boldsymbol{k}}(\boldsymbol{r})$ represents stationary waves adjusted to constrains included in the scattering matrix $S$. Two track solution can be written schematically as below, see Eq. (3).

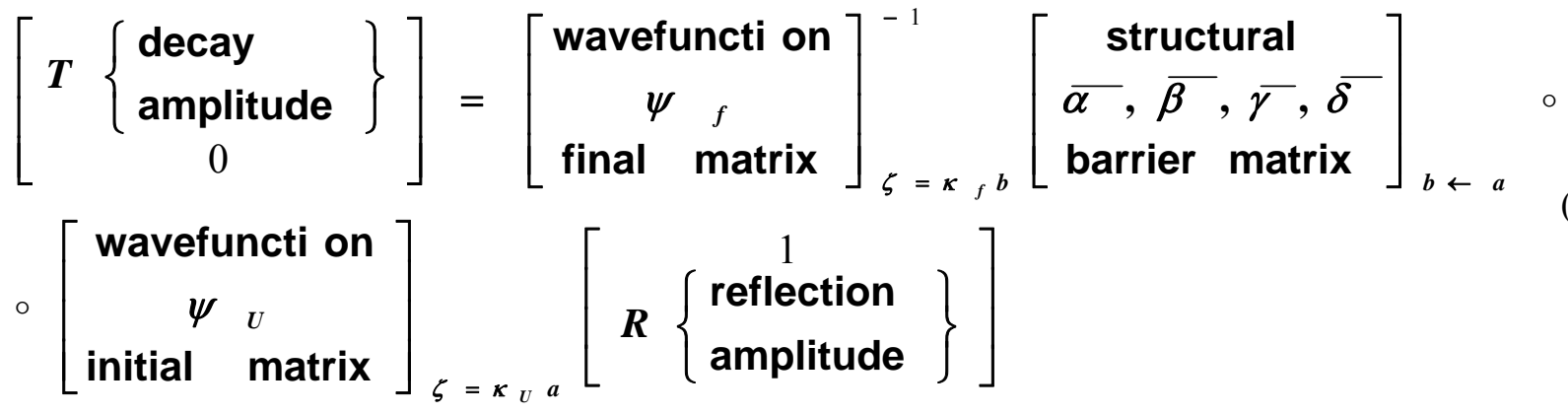

We can interpret matrix wave-functions $\left[\psi_{f}\right]$, $\left[\psi_{U}\right]$ according to Eq. (6) or (8). Generally speaking they are certain superposition of Wronskian and $U$ matrices.

The wave functions $\Psi_{f}=\left[\begin{array}{l}\psi_{f}^{\prime} \\ \psi_{f}\end{array}\right], \Psi_{B}=\left[\begin{array}{l}\psi^{\prime}{ }_{B} \\ \psi_{B}\end{array}\right]$, $\Psi_{U}=\left[\begin{array}{l}\psi_{U}^{\prime} \\ \psi_{U}\end{array}\right]$ are one column matrices with two rows. To understand tunneling effect we need both solutions-regular and irregular. The following question emerges: "If the wave function is not equal to zero at system origin, how such requirement might impact calculations?" In one dimension with two boundary points $a, b$ different from the system origin we have three waves: incoming $\psi$, reflected $\psi_{R}$ and transmitted $\psi_{T}$, and two output channels $(R, T)$ but only one phase shift $\eta_{l}$ for given partial wave. To include both mentioned channels we replace $S$-matrix by another unimodular $M$ matrix:

$$
\left[\begin{array}{c}
T \mathbf{H}^{i n w} \\
0
\end{array}\right]=[\boldsymbol{M}]\left[\begin{array}{c}
\mathbf{H}^{\text {inw }} \\
\mathbf{R} \mathbf{H}^{\text {outw }}
\end{array}\right]
$$

Comparing Eqs. (3) and (4) we deduce that the structural barrier matrix in Eq. (3) is proportional to $[M]$ matrix or in other words the structural matrix is included in $[M]$ matrix.

Such choice results in multipoint boundary conditions. The boundary conditions typical in all known codes (CRC-Coupled Reaction Channels, OM-Optical Model, CC-Coupled Channels, i.e. all DWUCK's, FRDWBA, LOLA, CHUCK's etc.) must be replaced by more general i.e. Dirichlet and von Neumann one (12).

$$
\begin{aligned}
& \alpha_{\psi} u(a)+\alpha_{\psi^{\prime}} u^{\prime}(a)=0 \\
& \beta_{\psi} u(b)+\beta_{\psi^{\prime}} u^{\prime}(b)=0
\end{aligned}
$$

The boundary continuity condition taken at $\boldsymbol{b}=\boldsymbol{r}_{0}$ according to the boundary condition (2) (i.e. where the internal solution is matched to the external one only in that point) can be formulated in terms of Wronskians. Matching generates the element $\boldsymbol{S}_{\boldsymbol{l}}$ of the scattering matrix:

$$
\begin{aligned}
& W\left\{\boldsymbol{\psi}_{I}^{(k)}, \mathbf{H}^{\text {inw }}\left(\boldsymbol{k} \boldsymbol{r}_{0}\right)+\boldsymbol{S}_{l} \mathbf{H}^{\text {outw }}\left(\boldsymbol{k} \boldsymbol{r}_{0}\right)\right\}= \\
& W\left\{\psi_{I}^{(k)}, \mathbf{H}^{i n w}\left(k r_{0}\right)\right\}+S_{I} \cdot W\left\{\psi_{I}^{(k)}, \mathbf{H}^{\text {outw }}\left(k r_{0}\right)\right\}=0 \\
& S_{I}=-\frac{W\left\{\psi_{I}^{(k)}, \mathbf{H}^{\text {inw }}\left(k r_{0}\right)\right\}}{W\left\{\psi_{I}^{(k)}, \mathbf{H}^{\text {outw }}\left(k r_{0}\right)\right\}}
\end{aligned}
$$

But when multiboundary conditions are applied we deal with the $M$ matrix built from $R, T$ amplitudes instead of dealing with the $S$ matrix. When $\boldsymbol{T} \rightarrow 0$ the amplitude $R$ changes into the $S$ matrix elements i.e. $\boldsymbol{R} \rightarrow \boldsymbol{S}$.

\section{Transmission-Tunneling through the Barrier}

To solve the transmission-tunneling problem-in other than Gamow's way-we take advantage of the 
Schrödinger equation matrix solutions $\left[\begin{array}{l}\psi_{f}^{\prime} \\ \psi_{f}\end{array}\right]$, $\left[\begin{array}{l}\psi_{B}^{\prime} \\ \psi_{B}\end{array}\right],\left[\begin{array}{l}\psi_{U}^{\prime} \\ \psi_{U}\end{array}\right]$ adequate for subsequent area of propagation. ( $f$-is the external area, $B$ - the barrier area, and $\mathrm{U}$ - the domain of solutions inside the well, see Fig. 1.

The column matrix in a given area consists of two elements. The lower element is the Schrödinger wave function and the derivative of $\boldsymbol{\psi}$ with respect to its argument is the upper one.

First we define: (c.f. [2]):

(a) The subsidiary $\boldsymbol{I}_{\boldsymbol{f}, \boldsymbol{B}}, \boldsymbol{I}_{\boldsymbol{B}, \boldsymbol{U}}$ matrices helpful in normalization of the $T$ amplitude $\boldsymbol{I}_{f, B}=\left[\begin{array}{cc}\sqrt{\frac{\kappa_{f}}{\kappa_{B}}} & 0 \\ 0 & \sqrt{\frac{\kappa_{f}}{\kappa_{B}}}\end{array}\right] ; \quad \boldsymbol{I}_{B, U}=\left[\begin{array}{cc}\sqrt{\frac{\kappa_{B}}{\kappa_{U}}} & 0 \\ 0 & \sqrt{\frac{\kappa_{B}}{\kappa_{U}}}\end{array}\right]$

(2) The interface $\mathbf{I}_{\boldsymbol{f}, \boldsymbol{B}}, \mathbf{I}_{\boldsymbol{B}, \boldsymbol{U}}$ matrices

$$
\begin{aligned}
\mathbf{I}_{f, B} & =\left[\begin{array}{cc}
\sqrt{\frac{\kappa_{f}}{\kappa_{B}}} & 0 \\
0 & \sqrt{\frac{\kappa_{B}}{\kappa_{f}}}
\end{array}\right]=\left[\begin{array}{cc}
\sqrt{\sigma_{f, B}} & 0 \\
0 & \frac{1}{\sqrt{\sigma_{f, B}}}
\end{array}\right] \\
\mathbf{I}_{B, U} & =\left[\begin{array}{cc}
\sqrt{\frac{\kappa_{B}}{\kappa_{U}}} & 0 \\
0 & \sqrt{\frac{\kappa_{U}}{\kappa_{B}}}
\end{array}\right]=\left[\begin{array}{cc}
\sqrt{\sigma_{B, U}} & 0 \\
0 & \frac{1}{\sqrt{\sigma_{B, U}}}
\end{array}\right]
\end{aligned}
$$

useful in matching solutions from the different domains

$$
\mathbf{I}_{f, B}\left[\begin{array}{l}
\psi_{f}^{\prime} \\
\psi_{f}
\end{array}\right]=I_{f, B}^{-1}\left[\begin{array}{l}
\psi_{B}^{\prime} \\
\psi_{B}
\end{array}\right] ; \quad \mathbf{I}_{B, U}\left[\begin{array}{l}
\psi_{B}^{\prime} \\
\psi_{B}
\end{array}\right]=I_{B, U}^{-1}\left[\begin{array}{l}
\psi_{U}^{\prime} \\
\psi_{U}
\end{array}\right]
$$

$\boldsymbol{\kappa}_{\boldsymbol{j}}, \boldsymbol{\sigma}_{\boldsymbol{j}, \boldsymbol{k}}$ etc. are explained in Appendix A.

These solutions can be written as:

$$
\begin{aligned}
& \mathbf{I}_{f, B}\left[\begin{array}{l}
\Psi_{f}^{\prime} \\
\Psi_{f}
\end{array}\right]_{\zeta=\kappa_{f} b}=I_{f, B}\left[\begin{array}{ll}
\mathbf{H}_{f, \text { in }}^{(1)}(\zeta) & \mathbf{H}_{f, \text { out }}^{(2)}(\zeta) \\
\mathbf{H}_{f, \text { in }}^{(1)}(\zeta) & \mathbf{H}_{f, \text { out }}^{(2)}(\zeta)
\end{array}\right]_{\zeta=\kappa_{f} b}\left[\begin{array}{c}
\bar{T} \\
0
\end{array}\right] \\
& I_{f, B}^{-1}\left[\begin{array}{l}
\Psi_{B}^{\prime} \\
\Psi_{B}
\end{array}\right]_{\zeta=\kappa_{B} b}=I_{f, B}^{-1}\left[\begin{array}{ll}
\psi_{B, \text { in }}^{(1)}(\zeta) & \psi_{B, \text { out }}^{(1)}(\zeta) \\
\psi_{B, \text { in }}^{(1)}(\zeta) & \psi_{B, \text { out }}^{(1)}(\zeta)
\end{array}\right]_{\zeta=\kappa_{B} b}\left[\begin{array}{c}
C \\
D
\end{array}\right]
\end{aligned}
$$

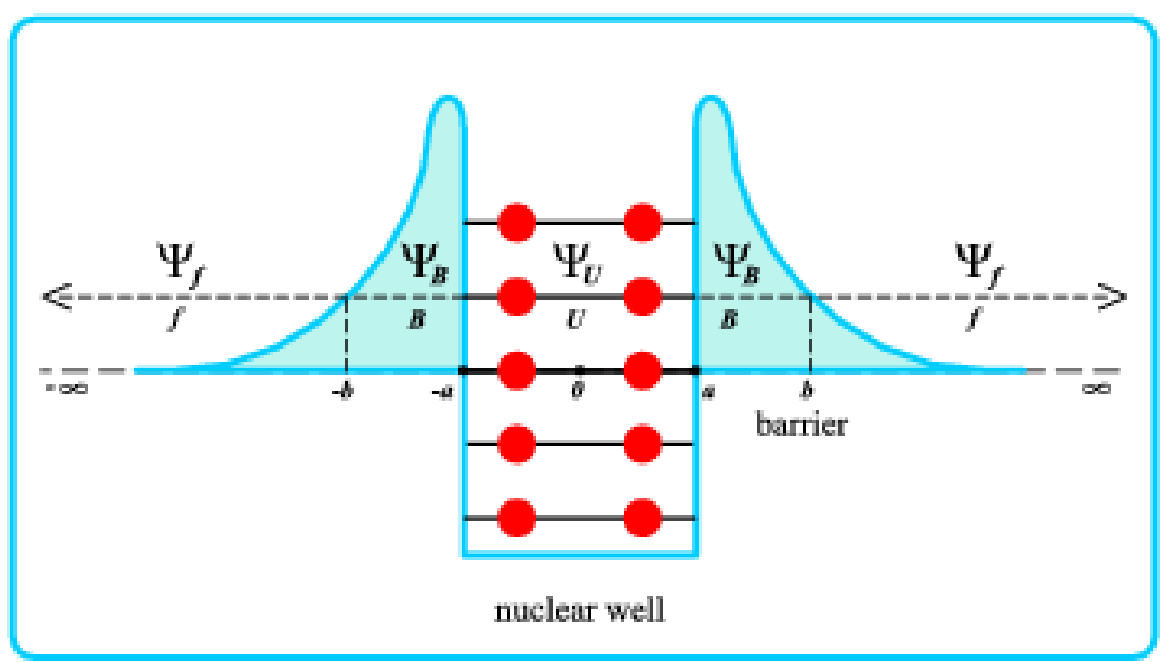

Fig. 1 Example of typical nuclear barrier applied to radioactivity decay with marked wave functions: external one- $\Psi_{f}$; under-barrier one $-\Psi_{B}$ and eigenfunction in the well $-\Psi_{U}$. We must apply the exact solution of the Schrödinger equation to each region. 


$$
\begin{aligned}
& \mathbf{I}_{B, U}\left[\begin{array}{l}
\psi_{B}^{\prime} \\
\psi_{B}
\end{array}\right]_{\zeta=\kappa_{B} a}=I_{B, U}^{-1}\left[\begin{array}{l}
\psi_{U}^{\prime} \\
\psi_{U}^{\prime}
\end{array}\right]_{\zeta=\kappa_{U} a} \\
& \mathbf{I}_{B, U}\left[\begin{array}{l}
\psi_{B}^{\prime} \\
\psi_{B}
\end{array}\right]_{\zeta=\kappa_{B} a}=\mathbf{I}_{B, U}\left[\begin{array}{ll}
\psi_{B, \text { in }}^{(1)}(\zeta) & \psi_{B, \text { out }}^{(1)}(\zeta) \\
\psi_{B, \text { in }}^{(1)}(\zeta) & \psi_{B, \text { out }}^{(1)}(\zeta)
\end{array}\right]_{\zeta=\kappa_{B} a}\left[\begin{array}{l}
C \\
D
\end{array}\right] \\
& \mathbf{I}_{B, U}^{-1}\left[\begin{array}{l}
\psi_{U}^{\prime} \\
\psi_{U}
\end{array}\right]_{\zeta=\kappa_{U} a}=\mathbf{I}_{B, U}^{-1}\left[\begin{array}{ll}
\mathbf{H}_{U, \text { in }}^{(1)}(\zeta) & \mathbf{H}_{U, \text { out }}^{(2)}(\zeta) \\
\mathbf{H}_{U, \text { in }}^{(1)}(\zeta) & \mathbf{H}_{U, \text { out }}^{(2)}(\zeta)
\end{array}\right]_{\zeta=\kappa_{U} a}\left[\begin{array}{l}
1 \\
R
\end{array}\right]
\end{aligned}
$$

In above equations we reveal following relations between $\psi_{j}$ on the left side and $\psi_{j, r}, \psi_{j, \text { ir }}$ or $\psi_{j, \text { in }}, \psi_{j, \text { out }}$ on the right side (where $\boldsymbol{j}=\{\boldsymbol{f}, \boldsymbol{B}, \boldsymbol{U}\}$ marks the intervals of the piecewise potential).

$$
\psi_{B}=f\left(\psi_{B, r}^{(1)}, \psi_{B, \text { ir }}^{(2)}\right)=f\left(\psi_{B, \text { in }}^{(1)}, \psi_{B, \text { out }}^{(2)}\right)
$$

The superscripts (1), (2) and/or the subscripts in, out (in case of inward and outward waves) or $r$, ir (in case of regular and irregular waves) can be omitted if we work with many typical mathematical functions as in Ref. [10].

$$
\psi_{B}=f\left(\psi_{B, r}, \psi_{B, \text { ir }}\right)=f\left(\psi_{B, \text { in }}, \psi_{B, \text { out }}\right)
$$

When we combine above five equations then the relation between $R$ and $T$ is:

$$
\begin{aligned}
& {\left[\begin{array}{c}
\overline{\boldsymbol{T}} \\
0
\end{array}\right]=\left[\begin{array}{cc}
\sqrt{\frac{\kappa_{U}}{\kappa_{f}}} & 0 \\
0 & \sqrt{\frac{\kappa_{U}}{\kappa_{f}}}
\end{array}\right]\left[\begin{array}{ll}
\mathbf{H}_{f, \text { in }}^{\prime(1)}(\zeta) & \mathbf{H}_{f, \text { out }}^{(2)}(\zeta) \\
\mathbf{H}_{f, \text { in }}^{(1)}(\zeta) & \mathbf{H}_{f, \text { out }}^{(2)}(\zeta)
\end{array}\right]_{\zeta=\boldsymbol{\kappa}_{f} b}^{-1}\left[\begin{array}{ll}
\bar{\delta} & \bar{\gamma} \\
\overline{\boldsymbol{\beta}} & \overline{\boldsymbol{\alpha}}
\end{array}\right]_{b \leftarrow a} \circ} \\
& {\left[\begin{array}{ll}
\mathbf{H}_{U, \text { in }}^{(1)}(\zeta) & \mathbf{H}_{U, \text { out }}^{(2)}(\zeta) \\
\mathbf{H}_{U, \text { in }}^{(1)}(\zeta) & \mathbf{H}_{U, \text { out }}^{(2)}(\zeta)
\end{array}\right]_{\zeta=\kappa_{U} a}\left[\begin{array}{c}
1 \\
\boldsymbol{R}
\end{array}\right]}
\end{aligned}
$$

Above equation can be schematically shown as:

$$
\begin{aligned}
& {\left[\begin{array}{c}
\overline{\boldsymbol{T}} \\
0
\end{array}\right]=\left[\begin{array}{c}
\text { normalization } \\
\boldsymbol{U} \rightarrow \boldsymbol{f}
\end{array}\right]\left[\begin{array}{c}
\text { wavefunction } \\
\text { final matrix }
\end{array}\right]_{\zeta=\kappa_{\boldsymbol{f}} b}^{-1}\left[\begin{array}{c}
\text { structural } \\
\text { barrier matrix }
\end{array}\right]_{b \leftarrow a} \circ} \\
& {\left[\begin{array}{l}
\text { wavefunction } \\
\text { initial matrix }
\end{array}\right]_{\zeta=\kappa_{U} a}\left[\begin{array}{c}
1 \\
\boldsymbol{R}
\end{array}\right]}
\end{aligned}
$$

Using $\sqrt{\frac{\kappa_{f}}{\kappa_{B}}} \overline{\boldsymbol{T}}=\boldsymbol{T}$ we get:

$$
\left[\begin{array}{c}
\boldsymbol{T} \\
0
\end{array}\right]=\left[\begin{array}{ll}
\mathbf{H}_{f, \text { in }}^{\prime(1)}(\zeta) & \mathbf{H}_{f, \text { out }}^{(2)}(\zeta) \\
\mathbf{H}_{f, \text { in }}^{(1)}(\zeta) & \mathbf{H}_{f, \text { out }}^{(2)}(\zeta)
\end{array}\right]_{\zeta=\kappa_{f} b}^{-1}\left[\begin{array}{ll}
\bar{\delta} & \bar{\gamma} \\
\bar{\beta} & \bar{\alpha}
\end{array}\right]_{b \leftarrow a}\left[\begin{array}{ll}
\mathbf{H}_{U, \text { in }}^{(1)}(\zeta) & \mathbf{H}_{U, \text { out }}^{(2)}(\zeta) \\
\mathbf{H}_{U, \text { in }}^{(1)}(\zeta) & \mathbf{H}_{U, \text { out }}^{(2)}(\zeta)
\end{array}\right]_{\zeta=\kappa_{U} a}\left[\begin{array}{c}
1 \\
\boldsymbol{R}
\end{array}\right]
$$

which looks like Eq. (3).

The under barrier transmission-reflection amplitudes between points $a$ and $b$ are given by the matrix called the structural barrier matrix. 


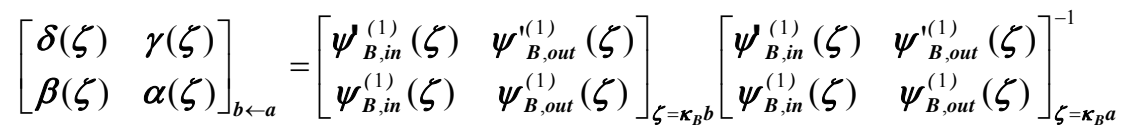

$$
\begin{aligned}
& =\left[\begin{array}{ll}
\psi_{B, r}^{(1)}(\zeta) & \psi_{B, i r}^{(1)}(\zeta) \\
\psi_{B, r}^{(1)}(\zeta) & \psi_{B, i r}^{(1)}(\zeta)
\end{array}\right]_{\zeta=\kappa_{B} b}[U][U]^{-1}\left[\begin{array}{ll}
\psi_{B, r}^{(1)}(\zeta) & \psi_{B, i r}^{(1)}(\zeta) \\
\psi_{B, r}^{(1)}(\zeta) & \psi_{B, i r}^{(1)}(\zeta)
\end{array}\right]_{\zeta=\kappa_{B} a}^{-1}
\end{aligned}
$$

That matrix propagates the Schrödinger equation solutions through the barrier (or the potential well).

The Wronskian matrix of the inward and outward wave-functions can also be expressed in terms of the regular and irregular wave-functions in the following way:

$$
\begin{aligned}
& {\left[\begin{array}{ll}
\psi_{B, \text { in }}^{(1)}(\zeta) & \psi_{B, \text { out }}^{(1)}(\zeta) \\
\psi_{B, \text { in }}^{(1)}(\zeta) & \psi_{B, \text { out }}^{(1)}(\zeta)
\end{array}\right]=\left[\begin{array}{ll}
\psi_{B, r}^{(1)}(\zeta) & \psi_{B, \text { ir }}^{(1)}(\zeta) \\
\psi_{B, r}^{(1)}(\zeta) & \psi_{B, i r}^{(1)}(\zeta)
\end{array}\right]_{\frac{1}{2}}\left[\begin{array}{cc}
i & -i \\
1 & 1
\end{array}\right]=} \\
& =\frac{1}{2}\left[\begin{array}{ll}
\psi_{B, \text { ir }}^{(1)}(\zeta)+i \psi_{B, r}^{(1)}(\zeta) & \psi_{B, \text { ir }}^{(1)}(\zeta)-i \psi_{B, r}^{(1)}(\zeta) \\
\psi_{B, i r}^{(1)}(\zeta)+i \psi_{B, r}^{(1)}(\zeta) & \psi_{B, i r}^{(1)}(\zeta)-i \psi_{B, r}^{(1)}(\zeta)
\end{array}\right]
\end{aligned}
$$

The total transmission-reflection matrix (through the barrier) when interface properties on both barrier sides are taken into account is given by

$$
\begin{aligned}
& {\left[\begin{array}{cc}
\bar{\delta} & \bar{\gamma} \\
\bar{\beta} & \bar{\alpha}
\end{array}\right]_{b \leftarrow a}=\left[\begin{array}{cc}
\sqrt{\frac{\kappa_{f}}{\kappa_{B}}} & 0 \\
0 & \sqrt{\frac{\kappa_{B}}{\kappa_{f}}}
\end{array}\right]^{-1}\left[\begin{array}{cc}
\delta(\zeta) & \gamma(\zeta) \\
\beta(\zeta) & \alpha(\zeta)
\end{array}\right]_{b \leftarrow a}\left[\begin{array}{cc}
\sqrt{\frac{\kappa_{B}}{\kappa_{U}}} & 0 \\
0 & \sqrt{\frac{\kappa_{U}}{\kappa_{B}}}
\end{array}\right]^{-1}=} \\
& {\left[\begin{array}{cc}
\sqrt{\frac{\kappa_{B}}{\kappa_{f}}} & 0 \\
0 & \sqrt{\frac{\kappa_{f}}{\kappa_{B}}}
\end{array}\right]\left[\begin{array}{ll}
\psi_{B, r}^{(1)}(\zeta) & \psi_{B, i r}^{(1)}(\zeta) \\
\psi_{B, r}^{(1)}(\zeta) & \psi_{B, i r}^{(1)}(\zeta)
\end{array}\right]_{\zeta=\kappa_{B} b}\left[\begin{array}{ll}
\psi_{B, r}^{(1)}(\zeta) & \psi_{B, i r}^{(1)}(\zeta) \\
\psi_{B, r}^{(1)}(\zeta) & \psi_{B, i r}^{(1)}(\zeta)
\end{array}\right]_{\zeta=\kappa_{B} a}^{-1}\left[\begin{array}{cc}
\sqrt{\frac{\kappa_{U}}{\kappa_{B}}} & 0 \\
0 & \sqrt{\frac{\kappa_{B}}{\kappa_{U}}}
\end{array}\right]}
\end{aligned}
$$

That matrix will be called the structural barrier matrix. The transmission $T$ and reflection $R$ are functions of elements of this matrix.

Eq. (6) can also be expressed by use of the regular and irregular waves $\mathbf{H}_{\boldsymbol{k}}=\boldsymbol{j}_{\boldsymbol{k}} \pm \boldsymbol{i n} \boldsymbol{k}_{\boldsymbol{k}}$

where $\boldsymbol{k} \in\{\boldsymbol{f}, \boldsymbol{B}, \boldsymbol{U}\}$ : (Similarity to the regular and irregular Bessel functions is kept $\left(\boldsymbol{\psi}_{\boldsymbol{k}}=\boldsymbol{u}_{\boldsymbol{k}} \pm \boldsymbol{i} \boldsymbol{v}_{\boldsymbol{k}}\right)$ in the function markings).

$$
\left[\begin{array}{l}
\boldsymbol{T} \\
0
\end{array}\right]=U^{-1}\left[\begin{array}{ll}
\boldsymbol{j}_{f}^{\prime}(\zeta) & \boldsymbol{n}_{f}^{\prime}(\zeta) \\
\boldsymbol{j}_{f}(\zeta) & n_{f}(\zeta)
\end{array}\right]_{\zeta=\kappa_{f} b}^{-1}\left[\begin{array}{ll}
\bar{\delta} & \bar{\gamma} \\
\bar{\beta} & \bar{\alpha}
\end{array}\right]_{b \leftarrow a}\left[\begin{array}{ll}
\boldsymbol{j}_{U}^{\prime}(\zeta) & \boldsymbol{n}_{U}^{\prime}(\zeta) \\
\boldsymbol{j}_{U}(\zeta) & \boldsymbol{n}_{U}(\zeta)
\end{array}\right]_{\zeta=\kappa_{U} a} \quad U\left[\begin{array}{l}
1 \\
R
\end{array}\right]
$$

Solving that equation we get:

(a) The transmission-tunneling decay amplitude through the barrier

$$
T=\frac{(\bar{\alpha} \bar{\delta}-\bar{\beta} \bar{\gamma})\left(\mathrm{H}^{\prime}{ }_{U}^{(1)}\left(\kappa_{U} a\right) \mathrm{H}_{U}^{(2)}\left(\kappa_{U} a\right)-\mathrm{H}_{U}^{(2)}\left(\kappa_{U} a\right) \mathrm{H}_{U}^{(1)}\left(\kappa_{U} a\right)\right)}{D E N O M}
$$

Where DENOM is:

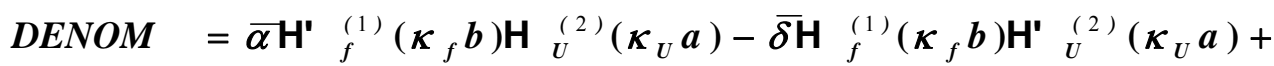

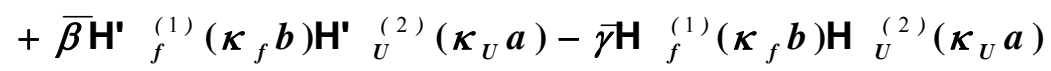

(b) The internal reflection amplitude from the barrier in the radioactive decay is: 


$$
\begin{aligned}
& \boldsymbol{R}((\bar{\delta}, \bar{\gamma}, \bar{\beta}, \bar{\alpha}), \boldsymbol{a}, \boldsymbol{b})=
\end{aligned}
$$

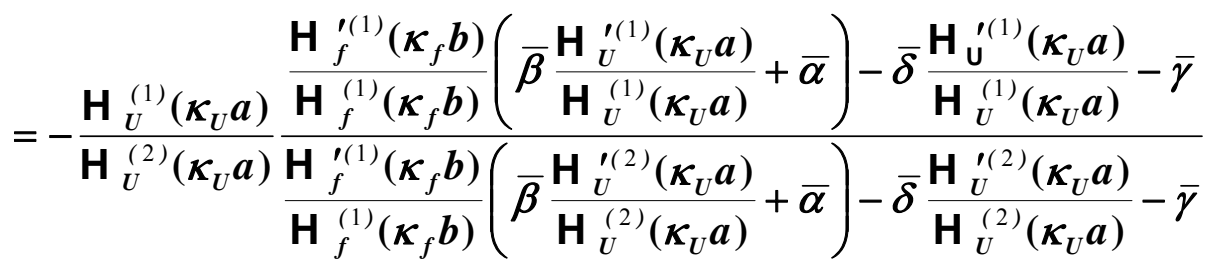

The notation illustrates the scattering on the square barrier. The barrier properties are included in $\bar{\delta}, \bar{\gamma}, \bar{\beta}, \bar{\alpha}$ coefficients. Of course $|\boldsymbol{R}| \leq 1$ and in $U$ region we can apply the eigenfunction (or wave-function) denoted by $\mathbf{H}_{U}$ or $\psi_{U}$, belonging to the level structure inside the potential well while in $f$ region we make use of the transmitted wave function given by solutions of Schrödinger equation outside the (nuclear) barrier.

When $\bar{\gamma}=\overline{\boldsymbol{\beta}}=0$ and $\overline{\boldsymbol{\delta}}=\overline{\boldsymbol{\alpha}}$ that amplitude $R$ reduces to

$$
\begin{aligned}
& \boldsymbol{R}((\overline{\boldsymbol{\delta}}=\overline{\boldsymbol{\alpha}}, \bar{\gamma}=\overline{\boldsymbol{\beta}}=0), \boldsymbol{a}, \boldsymbol{b})= \\
& \underline{\mathbf{H}_{f}^{\prime(1)}\left(\kappa_{f} b\right)}-\frac{\mathbf{H}_{U}^{\prime(1)}\left(\kappa_{U} a\right)}{H_{f}^{\prime(1)}\left(\kappa_{f} b\right)}-\frac{\mathbf{H}_{U}^{\prime(1)}\left(\kappa_{U} a\right)}{\psi_{f}^{(1)}\left(\kappa_{f} b\right)}
\end{aligned}
$$

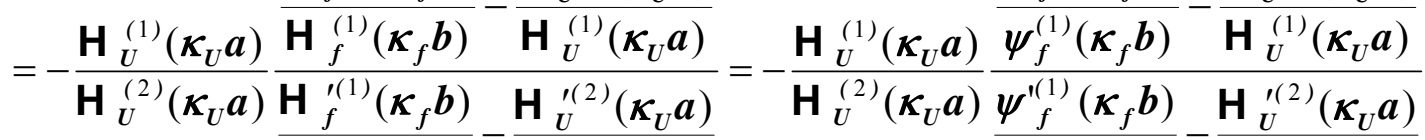

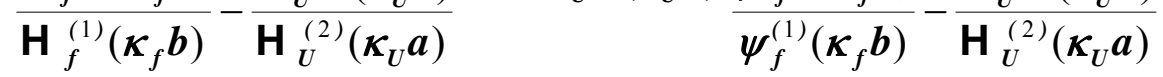

$$
\begin{aligned}
& R((\bar{\delta}=\bar{\alpha}, \bar{\gamma}=\bar{\beta}=0), a, b)=R(a, b)= \\
& -\frac{\psi_{f}^{\prime(1)}\left(\kappa_{f} b\right) \mathrm{H}_{U}^{(1)}\left(\kappa_{U} a\right)-\mathrm{H}_{U}^{\prime(1)}\left(\kappa_{U} a\right) \psi_{f}^{(1)}\left(\kappa_{f} b\right)}{\psi_{f}^{\prime(1)}\left(\kappa_{f} b\right) \mathrm{H}_{U}^{(2)}\left(\kappa_{U} a\right)-\mathrm{H}_{U}^{\prime(2)}\left(\kappa_{U} a\right) \psi_{f}^{(1)}\left(\kappa_{f} b\right)}
\end{aligned}
$$

If $a=b$ i.e. $R(b, b)=S$ matching takes place at the single point like in all standard codes:

$$
\boldsymbol{R}_{l}(b, b)=S_{I}=-\frac{W\left\{\psi_{l}^{(1)}\left(\kappa_{f} b\right), \mathbf{H}_{l}^{(1)}\left(\kappa_{U} b\right)\right\}}{W\left\{\psi_{l}^{(1)}\left(\kappa_{f} b\right), \mathbf{H}_{l}^{(2)}\left(\kappa_{U} b\right)\right\}}
$$

\section{Theory of Radioactive Decay, Classical} Gamow Theory of Decay versus Two Track Schrödinger Solutions

In this section a new approach to barrier transmission in radioactive decay is presented.

Separation energy of alpha particles of heavier atoms is negative, but it does not mean immediate decay of those nuclei. The experimentally measured particle decay energies lie below the barrier top. In the early stages of quantum mechanics not including barrier in calculations led to false interpretation that the charged particles impacted from outside interact with the Coulomb field but particles leaving the nucleus do not. Gamow theory of radioactive decay was one of the first successes in quantum mechanics.

The decay rate is proportional to the number $N$ of atoms and is expected in time interval $d t$ :

$$
\frac{d N}{d t}=-\lambda N \quad, \quad N=N(0) e^{-\lambda t}
$$

The proportionality constant $\lambda$ is calculated from available barrier transmission-tunneling models.

$$
\lambda=\lambda_{0}|\boldsymbol{T}|^{2}
$$

Usually $\lambda$ is given by the probability of transmission through the barrier between points $a$ and $b$, with given approximation according to WKB (Wentzel Kramers Brillouin). 


$$
|\boldsymbol{T}|^{2}=e^{-S}=e^{-2 \int_{a}^{b} \kappa(r) d r}
$$

is equal to frequency of well particle impacts on the barrier i.e. $1 / \tau=0 / 2 a$ where $2 a$ is internal diameter of the well.

To calculate the WKB integral $2 \int_{a}^{b} \kappa(r) d r$ we introduce quantities $V(\boldsymbol{r}), \eta_{C}$ defined as

$$
V(r)=\frac{Z_{\alpha} Z_{T} e^{2}}{r}
$$

$\eta_{C}=\frac{\boldsymbol{m}_{\alpha} \boldsymbol{Z}_{\alpha} \boldsymbol{Z}_{T}^{\boldsymbol{r}} \boldsymbol{e}^{2}}{\hbar^{2} \boldsymbol{k}}=\frac{\boldsymbol{Z}_{\alpha} \boldsymbol{Z}_{T} \boldsymbol{e}^{2}}{\hbar \boldsymbol{v}}$. Assuming equality

$$
S=\left.4 \eta_{C}\left(t-\frac{\sin (2 t)}{2}\right)\right|_{0} ^{\arccos \left(\sqrt{\frac{a}{b}}\right)}=
$$

Let us consider tunneling through the barrier-the problem launched by Gamow. Readers can compare Gamow solution with solution presented in Section 3. The similar comparison can be done according to Fig. 2 where the piecewise barrier is shown. In this case the structural barrier matrix (7) must be changed i.e. rebuilt by the multiple use of the matrix (7). It should be built according to our monodromy (i.e. multi-matching) procedure. Such piecewise structure enables to study influence of the barrier shape on the of the kinetic energy $\mathbf{E}$ of the $\alpha$ particle leaving the barrier and the energy released in the $\alpha$ decay i.e. $\boldsymbol{V}(\boldsymbol{b})=\mathbf{E}$, we can express point $\boldsymbol{b}=\frac{2 \eta_{C}}{\boldsymbol{k}}$ at which the particle is emitted. Hence the integral over $\boldsymbol{\kappa}(\boldsymbol{r})$ can be written as:

$$
2 \int_{a}^{b} \kappa(r) d r=2 \int_{a}^{b} \frac{1}{\hbar} \sqrt{V(r)-E} d r=2 \int_{x_{a}=a / b}^{x_{b}=1} \sqrt{\frac{1}{x}-1} d r
$$

After substitution $\boldsymbol{x}=\cos ^{2}(\boldsymbol{t})$ we get the final expression equal to the transmission exponent.

$$
=4 \eta_{C}\left[\arccos \left(\sqrt{\frac{a}{b}}\right)-\sqrt{\frac{a}{b}} \sqrt{1-\frac{a}{b}}\right]
$$

energy level structure in the well.

In the particle motion through the barriers as it is shown in Fig. 2 we mark off three regions denoted by suitable wave-functions: the well region with $\Psi_{U}$ the barrier one with $\Psi_{\boldsymbol{B}}$ and the external one with $\Psi_{f}$.

The Schrödinger solutions $\Psi_{U}$ in the potential well depend on the well shape. In the rectangular well case we can use the Bessel (or Hankel) functions to describe the particle motion inside it.

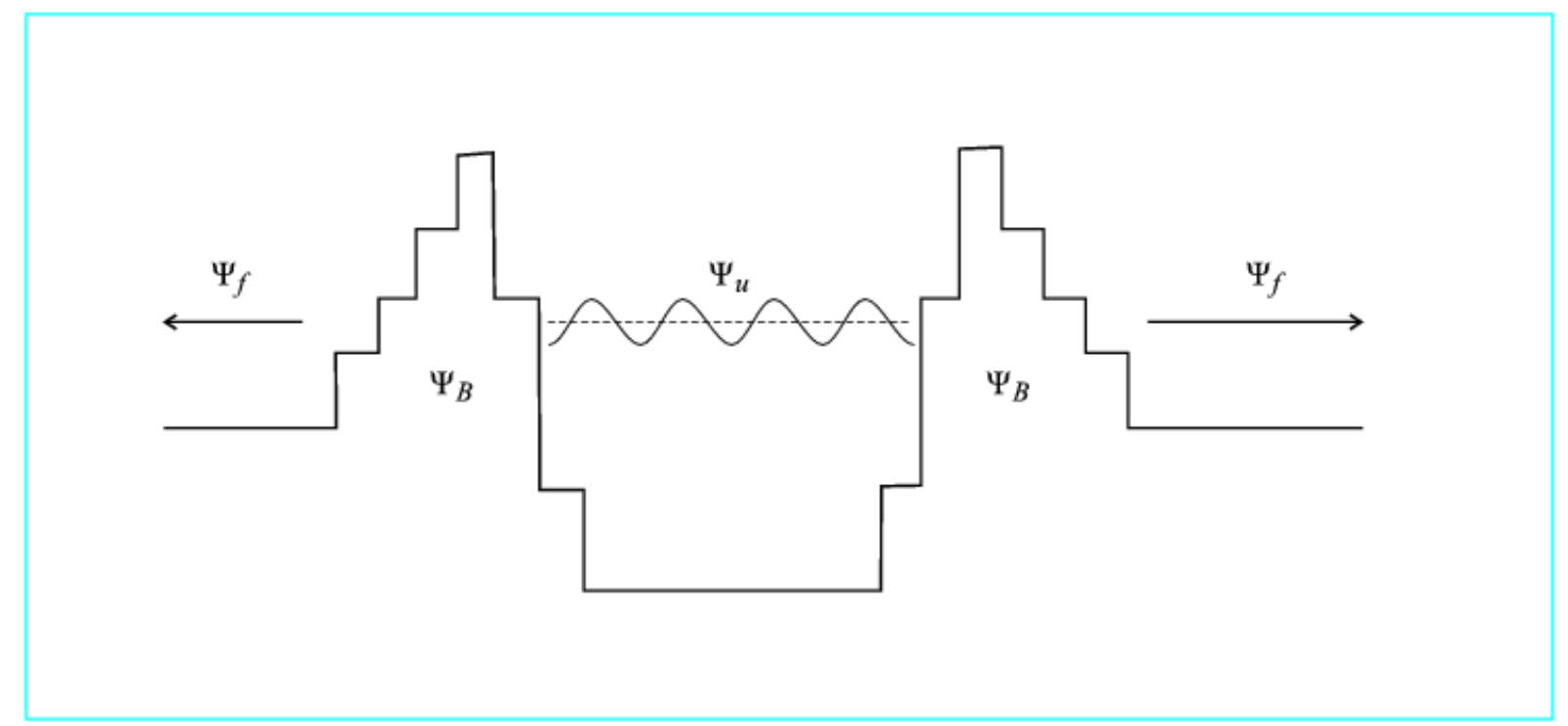

Fig. 2 Example of a piecewise nuclear barrier applied to radioactivity decay with marked wave-functions: external one$\Psi_{f}$; under-barrier one $-\Psi_{B}$ and eigenfunctionin the well- $\Psi_{U}$. Here monodromy requires the multiple matching of square barriers and/or the potential square wells. 
The wave-functions under the barrier $\Psi_{B}$, if the rectangular well shape is taken into account, are $I(\zeta), K(\zeta)$ where their argument is $\boldsymbol{\zeta}=\boldsymbol{\kappa}_{B} \boldsymbol{r}$.

The motion outside the nucleus is denoted by $\Psi_{f}$ and the Coulomb waves can be applied in that region. According to Eqs. (9) and (10) in Section 3 the transmission-tunneling $T$ amplitude can be written as:

$$
T=\frac{1}{\bar{\alpha} A_{I}-\bar{\delta} D_{I}+\bar{\beta} B_{I}-\bar{\gamma} C_{I}-i\left(\bar{\alpha} A_{R}-\bar{\delta} D_{R}+\bar{\beta} B_{R}-\bar{\gamma} C_{R}\right)}
$$

Comparing above equation with mentioned Eqs. (9) and (10) the new coefficients are

$$
\begin{aligned}
& A_{R}=\left(n_{f}^{\prime}\left(\kappa_{f} b\right) n_{U}\left(\kappa_{U} a\right)+j_{f}^{\prime}\left(\kappa_{f} b\right) j_{U}\left(\kappa_{U} a\right)\right) \\
& A_{I}=\left(j_{f}^{\prime}\left(\kappa_{f} b\right) n_{U}\left(\kappa_{U} a\right)-n_{f}^{\prime}\left(\kappa_{f} b\right) j_{U}\left(\kappa_{U} a\right)\right) \\
& D_{R}=\left(n_{f}\left(\kappa_{f} b\right) n_{U}^{\prime}\left(\kappa_{U} a\right)+j_{f}\left(\kappa_{f} b\right) j_{U}^{\prime}\left(\kappa_{U} a\right)\right) \\
& D_{I}=\left(j_{f}\left(\kappa_{f} b\right) n_{U}^{\prime}\left(\kappa_{U} a\right)-n_{f}\left(\kappa_{f} b\right) j_{U}^{\prime}\left(\kappa_{U} a\right)\right) \\
& B_{R}=\left(n_{f}^{\prime}\left(\kappa_{f} b\right) n_{U}^{\prime}\left(\kappa_{U} a\right)+j_{f}^{\prime}\left(\kappa_{f} b\right) j_{U}^{\prime}\left(\kappa_{U} a\right)\right) \\
& B_{I}=\left(j_{f}^{\prime}\left(\kappa_{f} b\right) n_{U}^{\prime}\left(\kappa_{U} a\right)-n_{f}^{\prime}\left(\kappa_{f} b\right) j_{U}^{\prime}\left(\kappa_{U} a\right)\right) \\
& C_{R}=\left(n_{f}\left(\kappa_{f} b\right) n_{U}\left(\kappa_{U} a\right)+j_{f}\left(\kappa_{f} b\right) j_{U}\left(\kappa_{U} a\right)\right) \\
& C_{I}=\left(j_{f}\left(\kappa_{f} b\right) n_{U}\left(\kappa_{U} a\right)-n_{f}\left(\kappa_{f} b\right) j_{U}\left(\kappa_{U} a\right)\right)
\end{aligned}
$$

The square barrier (for plane wave limits of above coefficients) requires substitutions:

$$
A_{R} \rightarrow 0, A_{I} \rightarrow 1, D_{R} \rightarrow 0, B_{R} \rightarrow 1, B_{I} \rightarrow 0, C_{R} \rightarrow 1, C_{I} \rightarrow 0
$$

Then we get:

$$
T=\frac{(\alpha \delta-\beta \gamma)}{\sqrt{\frac{\sigma_{\text {in }}}{\sigma_{f}}} \alpha+\sqrt{\frac{\sigma_{f}}{\sigma_{\text {in }}}} \delta-\frac{i}{\sqrt{\sigma_{i n} \sigma_{f}}} \beta+i \sqrt{\sigma_{i n} \sigma_{f} \gamma}}
$$

and the square of $T$ can be expressed as

$$
\frac{1}{|\boldsymbol{T}|^{2}}=\frac{\boldsymbol{\kappa}_{U}^{2}\left(1+\frac{\kappa_{f}}{\kappa_{U}}\right)^{2}}{4 \boldsymbol{\kappa}_{f} \boldsymbol{\kappa}_{U}}+\frac{\left(\frac{\kappa_{B}^{2}}{\kappa_{U}^{2}}-1\right)\left(1-\frac{\kappa_{f}^{2}}{\kappa_{B}^{2}}\right)}{4 \frac{\kappa_{f}}{\kappa_{U}}} \sin ^{2}\left(\boldsymbol{\kappa}_{B} \boldsymbol{d}\right)=\beta_{U \rightarrow f}+\alpha_{U \rightarrow f} \sin ^{2}\left(\boldsymbol{\kappa}_{B} \boldsymbol{d}\right)
$$

or

$$
\frac{1}{|\boldsymbol{T}|^{2}}=\frac{\left(1+\sigma_{f, U}\right)^{2}}{4 \sigma_{f, U}}+\frac{\left(\sigma_{B, U}^{2}-1\right)\left(1-\sigma_{f, B}^{2}\right)}{4 \sigma_{f, U}} \sin ^{2}\left(\boldsymbol{\kappa}_{B} \boldsymbol{d}\right)=\beta_{U \rightarrow f}+\alpha_{U \rightarrow f} \sin ^{2}\left(\boldsymbol{\kappa}_{B} \boldsymbol{d}\right)
$$

The Eq. (12) represents the most general formula of transmission-tunneling through any barrier system.

The $R, T$ amplitudes calculated from the matrix solutions of the time independent Schrödinger equation can be applied to the modified scattering theory to check transmission-tunneling influence on the cross sections as well as on other observables. In the same way we can check connection between the level structure in the potential well and the barrier shape.

Another application of our theory is presented in Ref. [2].

\section{Summary and Concluding Discussion}

In our paper we have studied boundary conditions influence on the Schrödinger equation solutions. The 


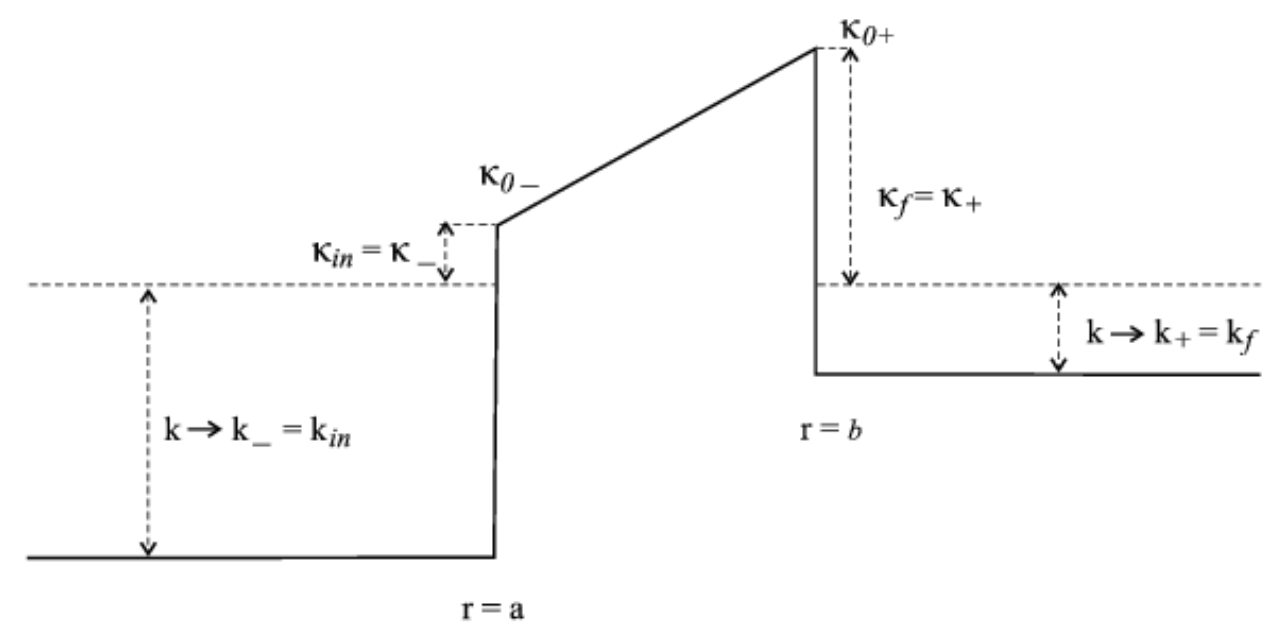

Fig. 3 The asymmetric barrier, the general case, the incoming particle momentum is $\hbar \boldsymbol{k}_{-}=\hbar \boldsymbol{k}_{\text {in }}$ the outgoing one is $\hbar \boldsymbol{k}_{+}=\hbar \boldsymbol{k}_{\boldsymbol{f}}, \boldsymbol{\kappa}_{0-}$ and $\boldsymbol{\kappa}_{0+}$ denote the wave numbers related to so called the reduced barrier height $\boldsymbol{\kappa}_{0 \pm}=\sqrt{2 \boldsymbol{m} \boldsymbol{V}_{0 \pm}} / \hbar$

standard boundary conditions (Eq. (2)), the wave function's equality to zero at the origin of the center mass system, and one-point matching condition cause lack of transmission and tunneling in the description of the observables measured in the scattering experiments. The optical theorem (as well as the unitarity relation in Ref. [6]) are derived within the framework of the partial wave analysis i.e. to each partial wave $\boldsymbol{\psi}_{\boldsymbol{l}, \overrightarrow{\boldsymbol{k}}}$ corresponds one phase shift $\boldsymbol{\eta}_{\boldsymbol{l}}$. There is no additional phase shift related to transmission or tunneling. Two additional phase shifts related to $\boldsymbol{S}_{\boldsymbol{I}}^{\boldsymbol{T}}, \boldsymbol{S}_{\boldsymbol{l}}^{(\boldsymbol{r t})}$ matrices appear in Section 1. These phase shifts contribute to ambiguity in phase shift analysis when they are not calculated directly.

In case of multi-point boundary value problem many methods are possible to calculate barrier superposition. These methods depend mainly on precise wave-function calculations in subdomains defined by given interval of the piecewise potential (c.f. Fig. 2) or in subdomains between any two subsequent matching points including a point at infinity (i.e. the intervals in Fig. 1 are: $(-\infty,-\boldsymbol{b})$, $(-\boldsymbol{b},-\boldsymbol{a})(-\infty,-\boldsymbol{b})(-\boldsymbol{a}, \boldsymbol{a})(\boldsymbol{a}, \boldsymbol{b})(\boldsymbol{b}, \infty)$; similar intervals can be found in Fig. 3. Two-point boundary condition shows that the scattering theory and the nuclear structure should not be investigated separately.

It is possible to study influence of the barrier shape on radioactive decays as well as on the level structure in the potential well when programs mentioned in Section 2 will be modified.

\section{Acknowledgments}

In remembrance of Prof. A. Lindner, the author of Refs. [8, 9], we would like to express our gratitude for his continued encouragement and support during the scientific stay in Hamburg of one of our team members in eighties and nineties.

\section{References}

[1] Grandy, W. T. 2000. Scattering of Waves from Large Spheres. Cambridge University Press.

[2] Jakiel, J., and Kantor, W. 2014. "Quantum (Not Frustrated) Theory of the Total Internal Reflection as the Source of the Goos-Hänchen Shift.” E. Phys. J. D68.

[3] Scott Carney, P., Schotland, J., and Wolf, E. 2004. "Generalized Optical Theorem for Reflection, Transmission, and Extinction of Power for Scalar Fields." Phys. Rev. E 70, 036611.

[4] Ramsauer, C. 1921. "Über den Wirkungsquerschnitt der Gasmoleküle gegenüber langsamen Elektronen.” 
Annalen der Physik 6 (64): 513.

[5] Townsend, J. S. 1921. "The Motion of Electrons in Gases." Philosophical Magazine S.6 (42): 873.

[6] Joachain, C. J. 1975. Collision Theory. Chap. Ch.4.4, Ch.11. North Holland Publishing Co.

[7] Melkanoff, M. A., Sawada, T., and Raynal, J. 1964. "Nuclear Optical Model Calculation." University of California, Los Angeles (unpublished).

[8] Lindner, A. 1984. Drehimpulse in der Quantenmechanik.
Chap. Ch.9, Ch.12. B. G. Teubner.

[9] Lindner, A. 1984. Grundkurs Theoretische Physik. Chap. Ch.3, Ch.5. B. G. Teubner.

[10] Abramowitz, M., and Stegun, I. A. 1964. Handbook of Mathematical Functions. National Bureau of Standards, 16 Applied Mathematics Series 55, Washington D.C. 20402.

[11] Messiah, A. 1961. Quantum Mechanics. Ch.III. North Holland Publishing Co. 


\section{Appendix A}

When we deal with the barrier consisting of $n$ square barriers, the domain of wave-function of Schrödinger equation must be divided into $n+2$ intervals. The incoming wave-function is defined on $j=0$ interval, the outgoing one is defined on the last one i.e. $j$ $=n+1$ interval. For the system of $j$-square barriers where $\boldsymbol{j} \in\{1,2, \ldots, \boldsymbol{n}\}$, the wave number $\boldsymbol{\kappa}_{0 \boldsymbol{j}}$ is so called the reduced j-barrier height $\left(\boldsymbol{\kappa}_{0 \boldsymbol{j}}=\sqrt{2 \boldsymbol{m} \boldsymbol{V}_{0 \boldsymbol{j}}} / \hbar\right)$. Then $\boldsymbol{\kappa}_{\boldsymbol{j}}=\sqrt{\boldsymbol{k}^{2}-\boldsymbol{\kappa}_{0 \boldsymbol{j}}^{2}}$ are the wave numbers in the subsequent intervals. The indexes $j=0$ and $j=\mathrm{n}+1$ define parts of the domain in front of barriers and outside them. For the same reference level at both sides of the barrier system (i.e. $\boldsymbol{\kappa}_{00}=\boldsymbol{\kappa}_{0, \boldsymbol{n}+1}=0$ ) we assume $\boldsymbol{k}=\boldsymbol{\kappa}_{0}=\boldsymbol{k}_{\boldsymbol{i n}}=\boldsymbol{k}_{\boldsymbol{f}}$.

For any barrier as based on Messiah textbook (11) we must define the reference level in regard to the incoming or outgoing particle momenta. For the single asymmetric barrier according to Fig. 3 we deal with the different reference energy level at both sides of the barrier. The incoming particle momentum is $\hbar \boldsymbol{k}_{\boldsymbol{i n}}=\hbar \boldsymbol{k}_{-}$and outgoing one is $\hbar \boldsymbol{k}_{\boldsymbol{f}}=\hbar \boldsymbol{k}_{+}$. The under-barrier wave numbers are $\boldsymbol{K}_{-}=\sqrt{\boldsymbol{k}_{-}^{2}-\boldsymbol{K}_{0-}^{2}}$ at one side and $\boldsymbol{\kappa}_{+}=\sqrt{\boldsymbol{k}_{+}^{2}-\boldsymbol{\kappa}_{0+}^{2}}$ the other barrier side. If we assume only one common reference level and all potential barriers are square then $\boldsymbol{k}_{-}=\boldsymbol{k}_{+}$and $\boldsymbol{\kappa}_{0 \boldsymbol{j}_{-}}=\boldsymbol{\kappa}_{0 \boldsymbol{j}_{+}}$. It is useful to introduce the quantities $\sigma_{-}=\sigma_{i n}=\frac{\kappa_{1}}{\boldsymbol{k}_{\text {in }}}$ and $\sigma_{+}=\sigma_{f}=\frac{\boldsymbol{\kappa}_{n}}{\boldsymbol{k}_{\text {in }}}$ describing the ratio of under-barrier wave number to incoming particle wave number. The quantity $\boldsymbol{\sigma}_{\boldsymbol{j}+1, \boldsymbol{j}}=\frac{\boldsymbol{\kappa}_{\boldsymbol{j}+1}}{\boldsymbol{\kappa}_{\boldsymbol{j}}}$ is the ratio of the wave numbers in the subsequent domains.

The prime (') denotes the derivative over the argument or wave-function phase i.e. $\boldsymbol{u}^{\prime}, \boldsymbol{v}^{\prime}$ means $\boldsymbol{u}^{\prime}(\boldsymbol{\zeta})=\boldsymbol{u}^{\prime}(\boldsymbol{k r})=\frac{\partial \boldsymbol{u}(\zeta)}{\partial \zeta}$

\section{Footnotes}

${ }^{1}$ According to Ref. [7] "the wave-function $\boldsymbol{u}_{1}$ must vanish at the origin and the asymptotic form of the solution should in principle be matched to a plane wave plus an outgoing wave."

2 The author of Refs. [8, 9] had introduced "einlaufende" and "auslaufende Coulombwellenfunktionen" denoted by Latin letters $I_{l}$ and $O_{l}$. In general case in our paper, any Schrödinger solutions are marked by $\psi_{\text {in }}=\psi_{r}+\mathbf{i} \psi_{\text {ir }}, \psi_{\text {out }}=\psi_{r}-\mathbf{i} \psi_{\text {ir }}$ while Hankel and/or Coulomb wave-function are denoted by $\mathbf{H}^{(1)}$ and $\mathbf{H}^{(2)}$. 